\title{
无金属参与下不饱和酰胺与多氯甲烷的串联环化反应
}

\author{
葛雅欣 $a$ 间芹芹 $a$ 田云飞 $*, b$ 王海军 $a$ 张春芳*,a 李泽江*,a \\ ( ${ }^{a}$ 河北大学化学与环境科学学院 药物化学与分子诊断教育部重点实验室 \\ 河北省化学生物学重点实验室 河北保定 071002) \\ ( $b$ 洛阳师范学院化学化工学院 河南省功能导向多孔材料重点实验室 河南洛阳 471934)
}

\begin{abstract}
摘要 发展了一类自由基引发不饱和酰胺与多氯甲烷的串联合环反应, 该体系提供了一种可选择性地生成多氯甲基取 代的异哇啉酮或二氢异喹啉酮的方法. 同时, 在该工作中开展了机理研究和光学实验.

关键词 不饱和酰胺; 多氯甲烷; 自由基反应; 异喹啉酮; 二氢异喹啉酮
\end{abstract}

\section{Metal-Free-Involved Cascade Cyclization of Unsaturated Amides with Polychloromethanes}

\author{
Ge, Yaxin ${ }^{a} \quad$ Yan, Qinqin $^{a} \quad$ Tian, Yunfei $^{*, b} \quad$ Wang, Haijun ${ }^{a}$ \\ Zhang, Chunfang*,a Li, Zejiang*,a \\ ( ${ }^{a}$ Key Laboratory of Medicinal Chemistry and Molecular Diagnosis of the Ministry of Education, Key Laboratory of Chemical \\ Biology of Hebei Province, College of Chemistry \& Environmental Science, Hebei University, Baoding, Hebei 071002) \\ ( ${ }^{b}$ Henan Key Laboratory of Function-Oriented Porous Materia, College of Chemistry and Chemical Engineering, \\ Luoyang Normal University, Luoyang, Henan 471934)
}

\begin{abstract}
Free radical initiated cascade cyclization of unsaturated amides with polychloromethanes provides a protocol for the selective synthesis of polychlorinated isoquinolinones, or dihydroisoquinolinones. A series of mechanistic studies and optical experiments were operated in this system.

Keywords unsaturated amide; polychloromethane; radical reaction; isoquinolinone; dihydroisoquinolinone
\end{abstract}

在过去几十年里, 大量具有生物、化学活性的多卤 代天然产物逐渐被人们发现并报道 ${ }^{[1-2]}$. 其中, 由于氯元 素可以方便地转化为羟基、氨基和羧基等常见官能团, 氯代烃类化合物被广泛用于有机合成中. 同时, 多氯代 有机化合物在医药和农药中具有重要的用途 ${ }^{[3]}$. 因此, 发展高效地合成多氯代有机化合物的体系已成为化学 家们的研究热点. 在 2005 年, Walsh 课题组 ${ }^{[4]}$ 报道了酶 催化下未活化脂肪类碳中心位点的卤代反应. 在 2010 年和 2011 年, Zakarian 课题组 ${ }^{[5]}$ 先后发展了二元 Ti-Ru 促进下 $N$-酰基噁唑烷酮类化合物可以发生选择性的氯 代反应. 最近, 自由基引发下烯烃与多氯甲烷的串联环
化反应分别被 $\mathrm{Loh}^{[6]}$, Zhao ${ }^{[7]}$, 及其他课题组 ${ }^{[8]}$ 报道. 尽 管该领域已被化学家们大量研究, 更加高效、简单的多 氯甲基化反应体系依旧有待探索.

由于异喹啉酮、二氢异喹啉酮类化合物具有很好的 生物、药物用途 ${ }^{[9-10]}$, 化学家们过去在合成以上两类氮 杂环化合物方面进行了大量研究. 其中, 异喹啉酮类化 合物主要是通过金属催化反应或氧化反应合成的 ${ }^{[1]}$, 相 关的自由基环化反应报道不多. 常见合成二氢异喹啉酮 类衍生物的方法包括酸、碱、过渡金属或自由基促进下 完成的反应体系 ${ }^{[12-14]}$.

鉴于多氯代有机化合物、氮杂环化合物的重要性,

\footnotetext{
* Corresponding authors. E-mail: lizejiang898@126.com; zhangcf@hbu.edu.cn; tianyunfly1120@163.com Received February 20, 2021; revised April 17, 2021; published online June 1, 2021.

Project supported by the National Natural Science Foundation of China (Nos. 21702044, 11704024, 21973024), the Natural Science Foundation of Hebei Province (Nos. B2020201014, B2020201006), the Science and Technology Project of Hebei Education Department (No. QN2019063), the Key Scientific Research Project of Higher Education of Henan Province (No. 21A150038) and the Natural Science Foundation of Henan Province (No. 162300410200). 国家自然科学基金(Nos. 21702044, 11704024, 21973024)、河北省自然科学基金(Nos. B2020201014, B2020201006)、河北省高等学校科学技术研究(No. QN2019063)、河南省高等学校重点科研(No. 21A150038)和河南省自然科学基金(No. 162300410200)资助项目.
} 
本工作尝试通过自由基策略合成多氯代异喹啉酮、二氢 异喹啉酮类衍生物. 尽管 Yu 课题组 ${ }^{[8 d]}$ 在 2020 年报道了 不饱和酰胺与多氯甲烷在过氧叔丁醚作用下发生串联 合环构建多氯代二氢异喹啉酮类化合物. 在课题组前期 有关自由基工作基础上 ${ }^{[15]}$, 研究发现改变多氯甲烷种 类, 可以选择性地分别制备多氯代异喹啉酮或二氢异喹 啉酮类化合物.

\section{1 结果与讨论}

\section{1 反应条件优化}

为了实现该类反应，以 $N$-甲基- $N$-烯丙基苯甲酰胺 (1a)和氯仿作为底物进行条件篮选(表 1). 首先, 发现改 变引发剂的种类和用量时, 过氧化二异丙苯(DCP)比过 氧叔丁醚(DTBP)、叔丁基过氧化异丙苯( TBCP)和过氧 苯甲酸叔丁酯(TBPB)效果好(Entries 1 7). 碘化钾作为 催化剂, 比 $N$-碘代丁二酰亚胺、碘化钠更有利于反应进 行(Entries 8 10). 接下来, 分别改变溶剂体积、反应时 间、温度都会不同程度影响目标产物收率(Entries 11 15). 最后, 发现在最佳条件下, 产物 2-甲基-4-(2,2,2-三 氯乙基)异喹啉-1(2H)-酮(2a)可以得到 $60 \%$ 的分离产率.

\section{2 底物适用范围考察}

在最优条件下, 课题组针对反应体系的底物耐受性 进行了考察(表 2). 首先, 研究了各种 $N$-烯丙基苯甲酰 胺与氯仿的反应情况. 由表 2 可见: 4-甲氧基(1b)、4-甲 基(1 c)、4-氟 $(\mathbf{1 d}) 、 4$-氯 $(\mathbf{1 e}) 、 4$-溴 $(\mathbf{1 f}) 、 4$-碘 $(\mathbf{1 g})$ 及 2 -萗 基(1h)取代的苯甲酰胺与氯仿在最佳条件下反应得到中 等收率的三氯甲基取代的异喹啉酮化合物( $\mathbf{2 b} \sim \mathbf{2 h}$ ). 接 下来, 发现 $N$-烯丙基- $N$ - 芐基苯甲酰胺、 $N$-烯丙基- $N-$ 乙 基苯甲酰胺和 $N$-烯丙基- $N$-丙基苯甲酰胺也可以与氯仿 发生反应生成 $33 \% \sim 72 \%$ 的相应异喹啉酮产物( $\mathbf{2} \mathbf{i} \sim 2 \mathbf{k}$ ). 令人惊讶的是, 各种 $N$-烯丙基苯甲酰胺与二氯甲烷在 相似的条件下反应时, 只生成了中等至较好收率的各种 二氯甲基取代的二氢异喹啉酮类衍生物, 并没有得到二 氯甲基取代的异喹啉酮类化合物(3a 3k)(表 3). 最后需 要说明的是, 由于电子效应、空间位阻效应, 吸电子及 邻位取代的苯甲酰胺不能与氯仿(或二氯甲烷)很好地发 生反应生成相应目标产物.

\section{3 反应机理研究}

为了探究反应机理, 进行了一系列控制实验 (Scheme 1), 首先, 氛代同位素实验(KIE)表明, 二氯甲 烷的碳氢键断裂为该反应的速控步(Scheme 1a). 自由基 钟实验验证了反应体系经历了自由基过程(Scheme 1b). 其次, 在反应体系中分别加入自由基抑制剂 2,2,6,6-四 甲基-1-哌啶氧化物(TEMPO)或 2,6-二叔丁基-4-甲基
表 1 反应条件优化 ${ }^{a}$

Table 1 Optimization of the typical conditions

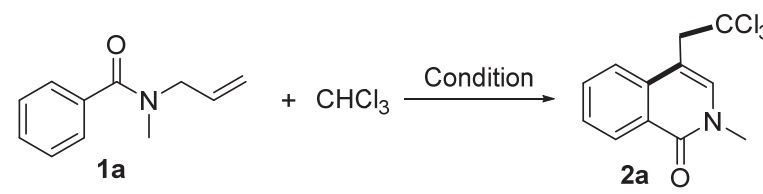

\begin{tabular}{ccccc}
\hline Entry & $\begin{array}{c}\text { Catalyst } \\
(\text { mol\%) }\end{array}$ & $\begin{array}{c}\text { Initiator } \\
\text { (equiv.) }\end{array}$ & Solvent (mL) & Yield $b \%$ \\
\hline 1 & KI (5) & DTBP (5.0) & Chloroform (7) & 37 \\
$\mathbf{2}$ & KI (5) & DCP (5.0) & Chloroform (7) & $\mathbf{6 0}$ \\
3 & KI (5) & TBCP (5.0) & Chloroform (7) & 49 \\
4 & KI (5) & TBPB (5.0) & Chloroform (7) & 39 \\
5 & KI (5) & DCP (3.0) & Chloroform (7) & 25 \\
6 & KI (5) & DCP (4.0) & Chloroform (7) & 32 \\
7 & KI (5) & DCP (6.0) & Chloroform (7) & 38 \\
8 & KI (10) & DCP (5.0) & Chloroform (7) & 33 \\
9 & NIS (5) & DCP (5.0) & Chloroform (7) & 46 \\
10 & NaI (5) & DCP (5.0) & Chloroform (7) & 50 \\
11 & KI (5) & DCP (5.0) & Chloroform (3) & 44 \\
12 & KI (5) & DCP (5.0) & Chloroform (5) & 38 \\
$13^{c}$ & KI (5) & DCP (5.0) & Chloroform (7) & 26 \\
$14^{d}$ & KI (5) & DCP (5.0) & Chloroform (7) & Trace \\
$15^{e}$ & KI (5) & DCP (5.0) & Chloroform (7) & Trace \\
\hline
\end{tabular}

${ }^{a}$ Reaction conditions: $N$-methyl- $N$-allylbenzamide (1 equiv., $0.1 \mathrm{mmol}$ ), chloroform $(7 \mathrm{~mL})$, KI ( 0.05 equiv., $0.005 \mathrm{mmol})$, DCP (5 equiv., $0.5 \mathrm{mmol})$, $130{ }^{\circ} \mathrm{C}, 24 \mathrm{~h} .{ }^{b}$ Isolated yield. ${ }^{c} 18 \mathrm{~h} .{ }^{d} 12 \mathrm{~h} .{ }^{e} 110{ }^{\circ} \mathrm{C}$.

苯酚(BHT)时，没有检测到目标产物 2a，同时，原料 1a 大量剩余; 此外, 通过气质联用仪检测到 TEMPO 捕获 甲基自由基的产物 1-甲氧基-2,2,6,6-四甲基哌啶(4b) (Scheme 1c). 最后, 研究发现底物 $N$-烯丙基- $N$-乙基苯 甲酰胺(1j) 在体系中反应 $24 \mathrm{~h}$ 后分离得到 44\%收率的二 氢异喹啉酮 $\mathbf{j} \mathbf{j}^{\prime}, \mathbf{2 j}$ '在最优条件下反应重新生成 74\%收率 的异喹啉酩 $\mathbf{2 j}$ (Scheme 1d).

经过以上实验验证, 给出了以下可能的反应机理 (Scheme 2). 首先, 在碘负离子作用下, 引发剂发生异 裂后生成氧中心自由基和氧负离子. 其中, 氧中心自由 基可能发生 $\beta$ 裂解生成甲基自由基. 多氯甲烷(氯仿或 二氯甲烷)被氧中心自由基或甲基自由基提取氢后生成 多氯甲基自由基，该自由基进攻 $N$-甲基- $N$-烯丙基苯甲 酰胺(1a)的双键后生成碳中心自由基 A. 中间体 $\mathbf{A}$ 合环 后生成芳基自由基 B. B 被氧化后生成芳基碳正离子 C. C 失去质子后生成多氯甲基取代的二氢异喹啉酩 D. 其 中, 三氯甲基取代的二氢异喹啉酮发生氢提取后生成自 由基 $\mathbf{E}$. E 经过两种途径(再次氢提取, 和先单电子氧化 生成 $\mathbf{F}$ 再失去质子)生成三氯甲基取代的异喹啉酮(2a). 通过两种模式的理论计算发现，三氯甲基取代的中间体 $\mathbf{D}$ 发生氢提取生成中间体 $\mathbf{E}$, 以及由 $\mathbf{E}$ 再次发生氢提取 生成异喹啉酮时释放的能量比二氯甲基取代的二氢异 
表 $2 N$-烯丙基苯甲酰胺与氯仿的串联环化反应 ${ }^{a}$

Table 2 Cascade cyclization of $\mathrm{N}$-allylbenzamides with chloroform

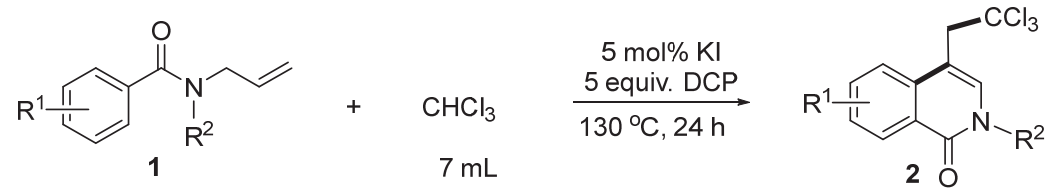

Substrate 12 Product $2 \quad$ Product 2

2a, $60 \%\left(20 \%^{c}\right)$<smiles>C=CCN(C)C(=O)c1ccc(OC)cc1</smiles><smiles>C=CCN(C)C(=O)c1ccc(C)cc1</smiles><smiles>C=CCN(C)C(=O)c1ccc(F)cc1</smiles><smiles>C=CCN(C)C(=O)c1ccc(Cl)cc1</smiles>

$1 e$<smiles>C=CCN(C)C(=O)c1ccc(Br)cc1</smiles><smiles>COc1ccc2c(=O)n(C)cc(CC(Cl)(Cl)Cl)c2c1</smiles>

2b, $65 \%$ (rsm 24\%)<smiles>Cc1ccc2c(=O)n(C)cc(CC(Cl)(Cl)Cl)c2c1</smiles>

2c, $52 \%(\mathrm{rsm} 43 \%)$<smiles>Cn1cc(CC(Cl)(Cl)Cl)c2cc(F)ccc2c1=O</smiles>

2d, $44 \%(\mathrm{rsm} 45 \%)$<smiles>Cn1cc(CC(Cl)(Cl)Cl)c2cc(Cl)ccc2c1=O</smiles><smiles>Cn1cc(CC(Cl)(Cl)Cl)c2cc(Br)ccc2c1=O</smiles>

2f, $38 \%($ rsm $47 \%)$

${ }^{a}$ Reaction conditions: $N$-allylbenzamides ( 1 equiv., $\left.0.1 \mathrm{mmol}\right)$, chloroform $(7 \mathrm{~mL})$, potassium iodide $(0.05 \mathrm{equiv} ., 0.005 \mathrm{mmol}), \mathrm{DCP}(5 \mathrm{equiv} ., 0.5 \mathrm{mmol}), 130{ }^{\circ} \mathrm{C}$, $24 \mathrm{~h}$, isolated yields, recovery of starting material (rsm) (in parentheses). ${ }^{b}$ Replenishing the same equivalent KI and DCP after $24 \mathrm{~h}$ in total $48 \mathrm{~h} .{ }^{c} \mathrm{Reaction}$ conditions: $N$-allylbenzamides ( 1 equiv., $5.7 \mathrm{mmol}, 1 \mathrm{~g})$, chloroform $(30 \mathrm{~mL})$, potassium iodide $(0.05$ equiv., $0.285 \mathrm{mmol}), \mathrm{DCP}(5 \mathrm{equiv} ., 28.5 \mathrm{mmol}), 130{ }^{\circ} \mathrm{C}, 24 \mathrm{~h}$, isolated yields.

喹啉酮要多. 因此，氯仿相对于二氯甲烷，更容易生成 异喹啉酮类化合物, 而二氯甲烷参与反应主要生成二氢 异喹啉酮类衍生物.

\section{4 应用研究}

接下来针对产物在光学方面的应用进行了研究(图 1). 产物 3-甲基-1-(2,2,2-三氯乙基)苯并[f]异喹啉-4(3H)- 
表 $3 \mathrm{~N}$-烯丙基苯甲酰胺与二氯甲烷的串联环化反应 ${ }^{a}$

Table 3 Cascade cyclization of $N$-allylbenzamides with dichloromethane<smiles>[R1]c1ccc(C(=O)N([R])CC=C)cc1</smiles><smiles>[R]N1CC(CCCl)C2=C(C=C[R1]C=C2)C1=O</smiles>

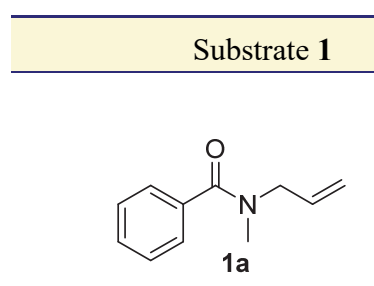<smiles>C=CCN(C)C(=O)c1ccc(OC)cc1</smiles>

1b<smiles>C=CCN(C)C(=O)c1ccc(C)cc1</smiles><smiles>C=CCN(C)C(=O)c1ccc(F)cc1</smiles><smiles>C=CCN(C)C(=O)c1ccc(Cl)cc1</smiles><smiles>C=CCN(C)C(=O)c1ccc(Br)cc1</smiles><smiles>CCCC1CN(C)C(=O)c2ccccc21</smiles>

3a, $70 \%$<smiles>COc1ccc2c(c1)C(CC(Cl)Cl)CN(C)C2=O</smiles>

3b, $42 \%$<smiles>COc1ccc2c(c1)C(CC(Cl)Cl)CN(C)C2=O</smiles>

3c, $70 \%$<smiles>CN1CC(CCCl)c2cc(F)ccc2C1=O</smiles>

3d, $46 \%$<smiles>CN1CC(CCCl)c2cc(Cl)ccc2C1=O</smiles>

3e, $65 \%$<smiles>CN1CC(CCCl)c2cc(Br)ccc2C1=O</smiles>

Substrate 1<smiles>C=CCN(C)C(=O)c1ccc(I)cc1</smiles><smiles>C=CCN(Cc1ccccc1)C(=O)c1ccccc1</smiles><smiles>C=CCN(CC)C(=O)c1ccccc1</smiles><smiles>C=CCN(CC)C(=O)c1ccccc1</smiles><smiles>C=CCN(C(=O)c1ccccc1)C1CCCC1</smiles>

$1 \mathrm{~m}$
Product 3<smiles>CN1CC(CCl)c2cc(I)ccc2C1=O</smiles>

$3 g, 55 \%$<smiles>O=C1c2ccccc2C(CC(Cl)Cl)CN1Cc1ccccc1</smiles>

3h, $60 \%$<smiles>CCN1CC(CCCl)c2ccccc2C1=O</smiles>

3i, $80 \%$<smiles>CCCN1CC(CCCl)c2ccccc2C1=O</smiles>

3j, $72 \%$<smiles>O=C1c2ccccc2C(CCCl)CN1C1CCCC1</smiles>

${ }^{a}$ Reaction conditions: $N$-allylbenzamides ( 1 equiv., $\left.0.1 \mathrm{mmol}\right)$, dichloromethane $(7 \mathrm{~mL})$, ammonium iodide $(0.05$ equiv., $0.005 \mathrm{mmol})$, TBPB (2 equiv., $\left.0.2 \mathrm{mmol}\right)$, $130{ }^{\circ} \mathrm{C}, 24 \mathrm{~h}$, isolated yields.

酮(2h)的紫外/可见吸收光谱(UV/Vis)显示: 在波长为 $325 \mathrm{~nm}$ 处有明显的吸收峰, 同时在波长为 352 和 $368 \mathrm{~nm}$ 处有两个肩峰. 此外, 产物 $\mathbf{2 h}$ 的激发光谱(PLE)表明: 在波长为 326,353 和 $367 \mathrm{~nm}$ 处有三个激发峰. 在波长为 $326 \mathrm{~nm}$ 光激发下, 得到了产物 $\mathbf{2 h}$ 的一个波长在 368 $518 \mathrm{~nm}$ 的发射光谱(PL), 其中, 最大发射峰波长为 411 $\mathrm{nm}$. 以上结果为该类产物在化学传感和有机光电材料 应用方面提供了一个参考.

\section{2 结论}

发展了一种自由基引发下 $N$-烯丙基苯甲酰胺与多 氯甲烷(氯仿和二氯甲烷)发生串联合环反应，并选择性 
(a) $\begin{array}{ll}\mathrm{CH}_{2} \mathrm{Cl}_{2} 3.5 \mathrm{~mL} & 5 \mathrm{~mol} \% \mathrm{NH}_{4} \mathrm{I} \\ & 2 \text { equiv. TBPB } \\ \mathrm{CD}_{2} \mathrm{Cl}_{2} 3.5 \mathrm{~mL} & 130^{\circ} \mathrm{C}, 10 \mathrm{~h}\end{array}$

1a

At low conversion $(16 \%), k_{\mathrm{H}} / k_{\mathrm{D}}=24$<smiles>CN1CC(CCCl)c2ccccc2C1=O</smiles><smiles>CN1CC(CC(=O)OCc2ccccc2)c2ccccc2C1=O</smiles>

(b)<smiles>C=CCN(C)CC=C</smiles>

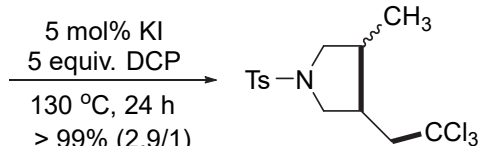

1n

$>99 \%(2.9 / 1)$

(c)<smiles>CON1C(C)(C)CCCC1(C)C</smiles>

$4 b$<smiles>Cn1cc(CC(Cl)(Cl)Cl)c2ccccc2c1=O</smiles>

2a, trace

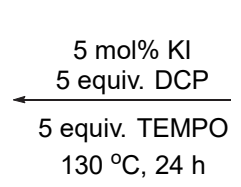
$130^{\circ} \mathrm{C}, 24 \mathrm{~h}$

$1 \mathrm{a}$

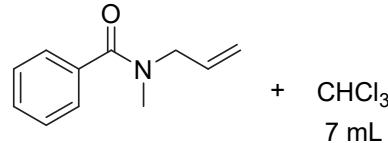

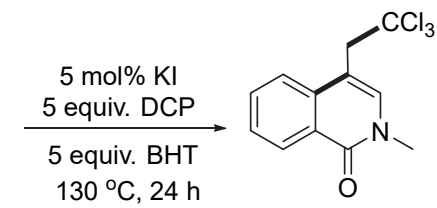

2a, trace

4b is detected by GC-MS. MS (EI) m/z (\%): 171 (5.9), 156 (100.0), 121 (38.4), 88 (24.2), 43 (46.7)

(d)<smiles>C=CCN(CC)C(=O)c1ccccc1</smiles>
$1 \mathrm{j}$

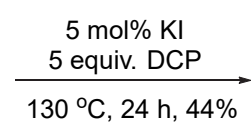<smiles>CCN1CC(CC(Cl)(Cl)Cl)c2ccccc2C1=O</smiles>

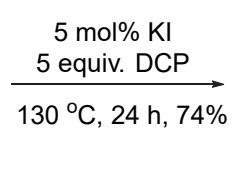<smiles>CCn1cc(CC(Cl)(Cl)Cl)c2ccccc2c1=O</smiles>

图式 $1 \mathrm{~N}$-烯丙基苯甲酰胺与多氯甲烷反应的控制实验

Scheme 1 Control experiments of the reactions of $N$-allylbenzamides with polychloromethanes

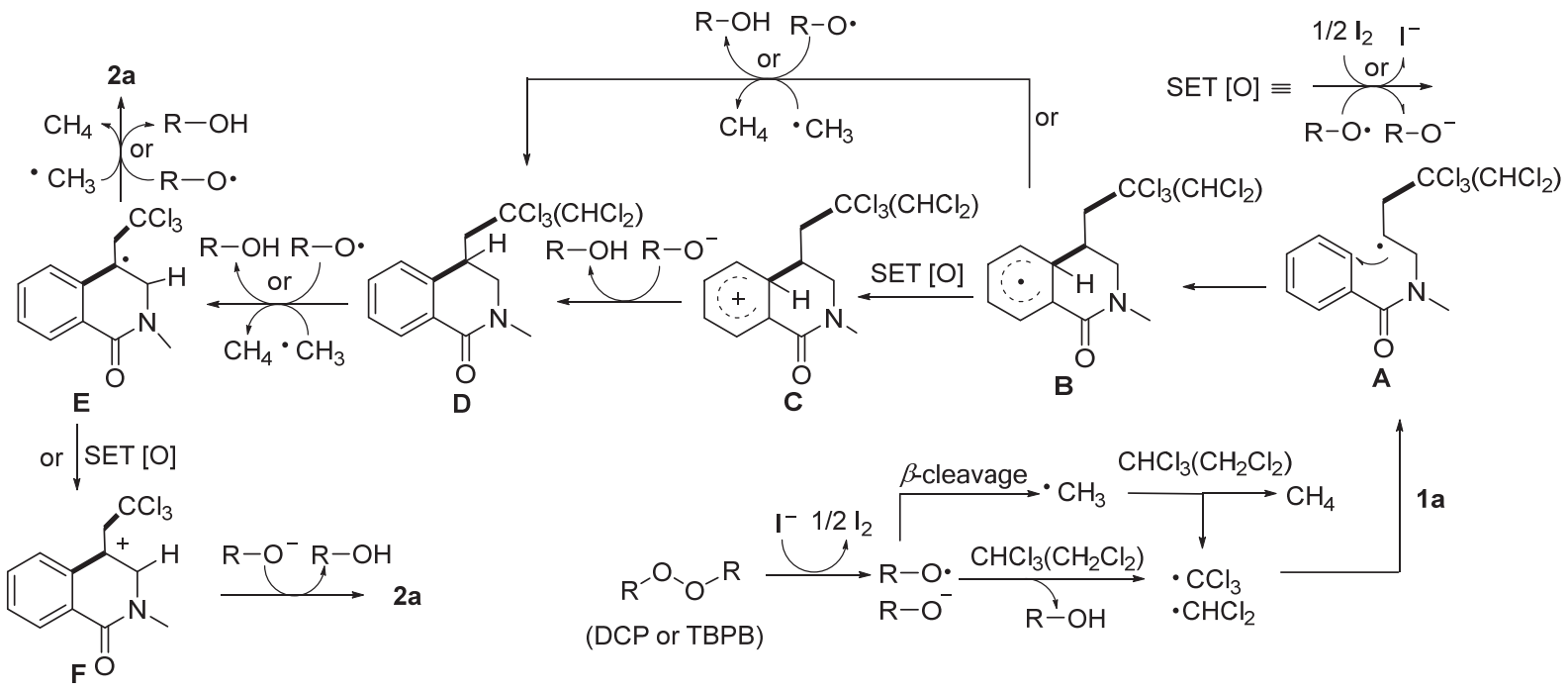

图式 $2 N$-烯丙基苯甲酰胺与多氯甲烷反应的可能机理

Scheme 2 Plausible mechanism of the reactions of $N$-allylbenzamides with polychloromethanes

地生成多氯甲基取代的异喹啉酮或二氢喹啉酮。此外, 对于该反应体系的可能机理和实际应用进行了部分研 究. 多氯甲烷与其它烯烃、炔烃的自由基串联反应在同 时开展之中.

\section{3 实验部分}

\section{1 仪器与试剂}

以気代氯仿作为溶剂, 使用 Bruker AVANCEIII 400 和 600 仪器测定了产物的核磁共振光谱; 以 Agilent
6520 或 Thermo QExactive 傅里叶变换离子回旋共振质 谱仪(ESI 源)测定了高分辨质谱; 用显微熔点测定仪(北 京泰克, X-4)测定了产物熔点, 温度未校正.

石油醚、乙酸乙酯购买于探索平台, 规格为 $5 \mathrm{~L}$ 铁 桶，未处理直接使用. 规格为 $200 \sim 300$ 目柱层析硅胶购 买于青岛海洋化工有限公司. 合成 $N$-烯丙基苯甲酰胺 的各种原料主要购买于安耐吉、迈瑞尔和 Alfa Aesar 等. 二氯甲烷和氯仿购买于科密欧. 所有试剂均未经处理直 接使用。 


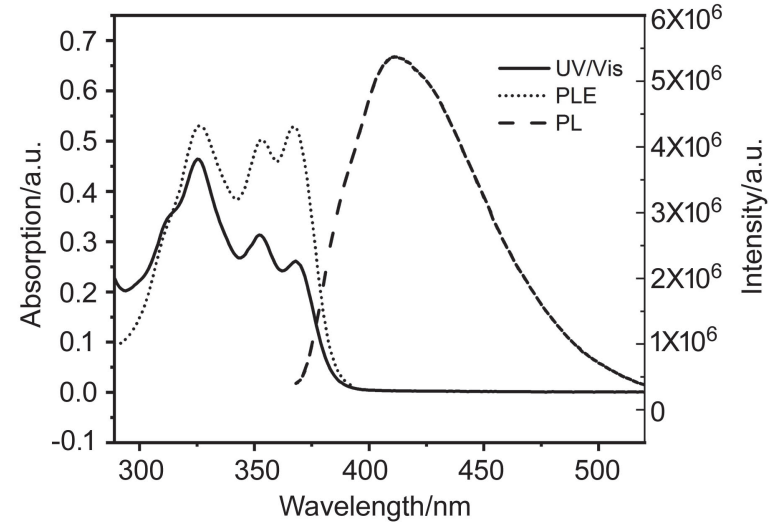

图 1 产物 $2 \mathrm{~h}$ 的光学研究

Figure 1 Optical study of product $\mathbf{2 h}$

UV/Vis absorption $\left(c=300 \mu \mathrm{mol} / \mathrm{L}\right.$ in $\left.\mathrm{CH}_{3} \mathrm{CN}\right)$, PLE spectra $(c=10 \mu \mathrm{mol} / \mathrm{L})$ and PL $(c=10 \mu \mathrm{mol} / \mathrm{L})$ of product $\mathbf{2 h}$

\section{2 实验方法}

\subsubsection{N-烯丙基苯甲酰胺的合成}

在 $50 \mathrm{~mL}$ 圆底烧瓶中依次加入各种芳基酰氯(1 equiv., $10 \mathrm{mmol}) 、$ 各种胺类(1 equiv., $10 \mathrm{mmol}) 、$ 二氯甲 烷 $(15 \mathrm{~mL})$ 和三乙胺 $(1.2$ equiv., $12 \mathrm{mmol})$, 一锅法在 $0{ }^{\circ} \mathrm{C}$ 反应 $3 \sim 10 \mathrm{~h}$. 通过薄层色谱检测反应完成后, 柱 层析得到各种芳基酰胺类化合物. 数据表征与文献[16] 一致.

在 $50 \mathrm{~mL}$ 圆底烧瓶中将上述芳基酰胺类(1 equiv., $10 \mathrm{mmol}$ )溶于 $10 \mathrm{~mL}$ 四氢呋喃中, $0{ }^{\circ} \mathrm{C}$ 下分批加入氢化 钠(1.1 equiv., $11 \mathrm{mmol})$, 冰水浴下继续搅拌 $0.5 \mathrm{~h}$, 将烯 丙基澳(1.1 equiv., $11 \mathrm{mmol}$ )滴加到反应混合物中, 移去 冰水浴并加入至 $80{ }^{\circ} \mathrm{C}$. 通过薄层色谱检测反应完成后, 柱层析得到各种 $N$-烯丙基苯甲酰胺类化合物.

\subsection{2 $\mathrm{N}$-烯丙基苯甲酰胺与氯仿或二氯甲烷的反应}

在 $25 \mathrm{~mL}$ 耐压管中依次加入 $N$-烯丙基苯甲酰胺 $(1$ equiv., $0.1 \mathrm{mmol})$ 、氯仿 $(7 \mathrm{~mL})$ 、碘化钾 $(0.05$ equiv., 0.005 $\mathrm{mmol})$ 和过氧化二异丙苯 (5 equiv., $0.5 \mathrm{mmol})$, 加热至 $130{ }^{\circ} \mathrm{C}$, 一锅法反应 $24 \mathrm{~h}$. 接下来通过薄层色谱检测反 应体系, 旋转蒸发仪浓缩反应混合物, 通过柱色谱分离 得到三氯甲基取代的异喹啉酮类化合物。

在 $25 \mathrm{~mL}$ 耐压管中依次加入 $N$-烯丙基苯甲酰胺 (1 equiv., $0.1 \mathrm{mmol})$, 二氯甲烷 $(7 \mathrm{~mL})$, 碘化铵 $(0.05$ equiv., $0.005 \mathrm{mmol})$, 过氧苯甲酸叔丁酯 $(2$ equiv., $0.2 \mathrm{mmol})$, 加热至 $130{ }^{\circ} \mathrm{C}$ 后反应 $24 \mathrm{~h}$. 通过上述相同操作得到二氯 甲基取代的二氢异喹啉酮类衍生物.

\section{3 化合物表征}

2-甲基-4-(2,2,2-三氯乙基)异喹啉-1(2H)-酮(2a)：黄 色固体, 产率 60\%. m.p. $145 \sim 146{ }^{\circ} \mathrm{C} ;{ }^{1} \mathrm{H}$ NMR (400 $\left.\mathrm{MHz}, \mathrm{CDCl}_{3}\right) \delta: 8.50 \sim 8.48(\mathrm{~m}, 1 \mathrm{H}), 7.83(\mathrm{~d}, J=8.0 \mathrm{~Hz}$,
1H), $7.71 \sim 7.67(\mathrm{~m}, 1 \mathrm{H}), 7.50(\mathrm{t}, J=7.6 \mathrm{~Hz}, 1 \mathrm{H}), 7.30(\mathrm{~s}$, 1H), 4.06 (s, 2H), 3.65 (s, 3H); ${ }^{13} \mathrm{C}$ NMR $(100 \mathrm{MHz}$, $\left.\mathrm{CDCl}_{3}\right) \delta: 162.1,136.5,136.1,131.9,128.2,126.8,125.9$, 123.5, 108.5, 99.3, 52.4, 37.3. HRMS (ESI) calcd for $\mathrm{C}_{12} \mathrm{H}_{11} \mathrm{Cl}_{3} \mathrm{NO}[\mathrm{M}+\mathrm{H}]^{+}$289.9901, found 289.9900.

6-甲氧基-2-甲基-4-(2,2,2-三氯乙基)异喹啉-1(2H)酮(2b): 黄色液体, 产率 65\% (rsm 24\%). ${ }^{1} \mathrm{H}$ NMR (400 $\left.\mathrm{MHz}, \mathrm{CDCl}_{3}\right) \delta: 8.41(\mathrm{~d}, J=9.2 \mathrm{~Hz}, 1 \mathrm{H}), 7.28(\mathrm{~s}, 1 \mathrm{H})$, $7.17(\mathrm{~d}, J=2.4 \mathrm{~Hz}, 1 \mathrm{H}), 7.08(\mathrm{dd}, J=8.8,2.4 \mathrm{~Hz}, 1 \mathrm{H})$, $4.02(\mathrm{~s}, 2 \mathrm{H}), 3.93$ (s, 3H), 3.63 (s, 3H); ${ }^{13} \mathrm{C}$ NMR $(100$ $\left.\mathrm{MHz}, \mathrm{CDCl}_{3}\right) \delta: 162.4,161.9,138.6,137.0,130.4,119.9$, 115.4, 108.1, 105.8, 99.4, 55.5, 52.7, 37.1. HRMS (ESI) calcd for $\mathrm{C}_{13} \mathrm{H}_{13} \mathrm{Cl}_{3} \mathrm{NO}_{2}[\mathrm{M}+\mathrm{H}]^{+} 320.0006$, found 320.0009 .

2,6-二甲基-4-(2,2,2-三氯乙基)异喹啉-1(2H)-酮(2c): 白色固体, 产率 $52 \%$ (rsm 43\%). m.p. 81 82 ${ }^{\circ} \mathrm{C} ;{ }^{1} \mathrm{H}$ NMR (400 MHz, $\left.\mathrm{CDCl}_{3}\right) \delta: 8.37(\mathrm{~d}, J=8.4 \mathrm{~Hz}, 1 \mathrm{H}), 7.58$ (s, 1H), 7.32 (d, $J=8.4 \mathrm{~Hz}, 1 \mathrm{H}), 7.28(\mathrm{~s}, 1 \mathrm{H}), 4.05(\mathrm{~s}, 2 \mathrm{H})$, $3.64(\mathrm{~s}, 3 \mathrm{H}), 2.51(\mathrm{~s}, 3 \mathrm{H}) ;{ }^{13} \mathrm{C}$ NMR $\left(100 \mathrm{MHz}, \mathrm{CDCl}_{3}\right) \delta$ : $162.1,142.4,136.7,136.2,128.5,128.2,123.8,123.3$, 108.3, 99.4, 52.4, 37.2, 22.1. HRMS (ESI) calcd for $\mathrm{C}_{13} \mathrm{H}_{13} \mathrm{Cl}_{3} \mathrm{NO}[\mathrm{M}+\mathrm{H}]^{+}$304.0057, found 304.0057.

6-氟-2-甲基-4-(2,2,2-三氯乙基)异喹啉-1(2H)-酮 (2d): 白色固体, 产率 44\% (rsm 45\%). m.p. 134 $135{ }^{\circ} \mathrm{C} ;{ }^{1} \mathrm{H}$ NMR $\left(400 \mathrm{MHz}, \mathrm{CDCl}_{3}\right) \delta: 8.50$ (dd, $J=8.8$, $6.0 \mathrm{~Hz}, 1 \mathrm{H}), 7.45$ (dd, $J=10.4,2.4 \mathrm{~Hz}, 1 \mathrm{H}), 7.34$ (s, 1H), 7.21 (td, $J=8.4,2.4 \mathrm{~Hz}, 1 \mathrm{H}), 4.00$ (s, 2H), 3.64 (s, 3H); ${ }^{13} \mathrm{C}$ NMR $\left(100 \mathrm{MHz}, \mathrm{CDCl}_{3}\right) \delta: 166.4,162.7$ (d, $J=239.6$ $\mathrm{Hz}), 139.1$ (d, $J=9.9 \mathrm{~Hz}), 137.5,131.5(\mathrm{~d}, J=10.0 \mathrm{~Hz})$, 122.58 (d, $J=1.8 \mathrm{~Hz}), 115.5$ (d, $J=23.3 \mathrm{~Hz}), 109.0$ (d, $J=$ $23.3 \mathrm{~Hz}), 107.9$ (d, $J=3.3 \mathrm{~Hz}$ ), 99.0, 52.6, 37.3; ${ }^{19} \mathrm{~F}$ NMR $\left(565 \mathrm{MHz}, \mathrm{CDCl}_{3}\right) \delta$ : -105.79 (s, 1F); HRMS (ESI) calcd for $\mathrm{C}_{12} \mathrm{H}_{10} \mathrm{Cl}_{3} \mathrm{FNO}[\mathrm{M}+\mathrm{H}]^{+}$307.9807, found 307.9808 .

6-氯-2-甲基-4-(2,2,2-三氯乙基)异喹啉-1(2H)-酮 (2e): 白色固体，产率 52\% (rsm 40\%). m.p. 114 $115{ }^{\circ} \mathrm{C} ;{ }^{1} \mathrm{H}$ NMR $\left(400 \mathrm{MHz}, \mathrm{CDCl}_{3}\right) \delta: 8.42(\mathrm{~d}, J=8.8$ $\mathrm{Hz}, 1 \mathrm{H}), 7.79(\mathrm{~d}, J=1.6 \mathrm{~Hz}, 1 \mathrm{H}), 7.45(\mathrm{dd}, J=8.4,1.6 \mathrm{~Hz}$, $1 \mathrm{H}), 7.33(\mathrm{~s}, 1 \mathrm{H}), 4.01(\mathrm{~s}, 2 \mathrm{H}), 3.64(\mathrm{~s}, 3 \mathrm{H}) ;{ }^{13} \mathrm{C} \mathrm{NMR}$ $\left(100 \mathrm{MHz}, \mathrm{CDCl}_{3}\right) \delta: 161.5,138.8,138.0,137.5,130.1$, 127.4, 124.3, 123.1, 107.6, 99.0, 52.4, 37.3. HRMS (ESI) calcd for $\mathrm{C}_{12} \mathrm{H}_{10} \mathrm{Cl}_{4} \mathrm{NO}[\mathrm{M}+\mathrm{H}]^{+}$323.9511, found 323.9513 .

6-溴-2-甲基-4-(2,2,2-三氯乙基)异喹啉-1(2H)-酮 (2f): 黄色固体, 产率 38\% (rsm 47\%). m.p. 164 165 ${ }^{\circ} \mathrm{C}$; 
${ }^{1} \mathrm{H}$ NMR (400 MHz, $\left.\mathrm{CDCl}_{3}\right) \delta: 8.33(\mathrm{~d}, J=8.8 \mathrm{~Hz}, 1 \mathrm{H})$, $7.96(\mathrm{~d}, J=1.6 \mathrm{~Hz}, 1 \mathrm{H}), 7.60$ (dd, $J=8.4,1.6 \mathrm{~Hz}, 1 \mathrm{H})$, 7.33 (s, 1H), $4.01(\mathrm{~s}, 2 \mathrm{H}), 3.64(\mathrm{~s}, 3 \mathrm{H}) ;{ }^{13} \mathrm{C}$ NMR $(100$ $\left.\mathrm{MHz}, \mathrm{CDCl}_{3}\right) \delta: 161.7,138.1,137.5,130.2,130.1,127.5$, 126.3, 124.6, 107.5, 99.0, 52.3, 37.4. HRMS (ESI) calcd for $\mathrm{C}_{12} \mathrm{H}_{10} \mathrm{BrCl}_{3} \mathrm{NO}[\mathrm{M}+\mathrm{H}]^{+}$367.9006, found 367.9009.

6-碘-2-甲基-4-(2,2,2-三氯乙基)异喹啉-1(2H)-酮 (2g): 白色固体, 产率 36\% (rsm 47\%). m.p. 151 $152{ }^{\circ} \mathrm{C} ;{ }^{1} \mathrm{H}$ NMR $\left(400 \mathrm{MHz}, \mathrm{CDCl}_{3}\right) \delta: 8.16(\mathrm{~d}, J=8.4$ $\mathrm{Hz}, 2 \mathrm{H}), 7.80$ (dd, $J=8.4,1.6 \mathrm{~Hz}, 1 \mathrm{H}), 7.31$ (s, $1 \mathrm{H}), 4.00$ (s, 2H), 3.63 (s, 3H). ${ }^{13} \mathrm{C}$ NMR (100 MHz, $\left.\mathrm{CDCl}_{3}\right) \delta$ : $161.8,138.0,137.3,135.9,132.6,129.8,125.1,107.2$, 100.2, 99.0, 52.3, 37.4. HRMS (ESI) calcd for $\mathrm{C}_{12} \mathrm{H}_{10} \mathrm{Cl}_{3} \mathrm{INO}[\mathrm{M}+\mathrm{H}]^{+}$415.8867, found 415.8866.

3-甲基-1-(2,2,2-三氯乙基)苯并 $[f$ 异喹啉-4(3H)-酮 (2h): 黄色液体, 产率 32\%. ${ }^{1} \mathrm{H}$ NMR $\left(400 \mathrm{MHz}, \mathrm{CDCl}_{3}\right)$ $\delta: 8.67(\mathrm{~d}, J=7.6 \mathrm{~Hz}, 1 \mathrm{H}), 8.49(\mathrm{~d}, J=8.8 \mathrm{~Hz}, 1 \mathrm{H}), 7.95 \sim$ $7.93(\mathrm{~m}, 1 \mathrm{H}), 7.86(\mathrm{~d}, J=8.8 \mathrm{~Hz}, 1 \mathrm{H}), 7.65 \sim 7.62(\mathrm{~m}$, 2H), 7.49 (s, 1H), 4.67 (s, 2H), 3.73 (s, 3H); ${ }^{13} \mathrm{C}$ NMR $\left(100 \mathrm{MHz}, \mathrm{CDCl}_{3}\right) \delta: 166.0,138.1,136.1,135.6,129.4$, $129.2,128.4,127.8,126.9,126.0,125.1,124.1,108.9$, 99.8, 56.4, 37.3. HRMS (ESI) calcd for $\mathrm{C}_{16} \mathrm{H}_{13} \mathrm{Cl}_{3} \mathrm{NO}$ $[\mathrm{M}+\mathrm{H}]^{+} 340.0057$, found 340.0059 .

2-芐基-4-(2,2,2-三氯乙基)异喹啉-1(2H)-酮(2i): 黄 色液体, 产率 $58 \% .{ }^{1} \mathrm{H}$ NMR $\left(400 \mathrm{MHz}, \mathrm{CDCl}_{3}\right) \delta: 8.53$ (dd, $J=8.0,1.2 \mathrm{~Hz}, 1 \mathrm{H}), 7.83(\mathrm{~d}, J=8.0 \mathrm{~Hz}, 1 \mathrm{H}), 7.72 \sim$ $7.68(\mathrm{~m}, 1 \mathrm{H}), 7.52(\mathrm{t}, J=7.2 \mathrm{~Hz}, 1 \mathrm{H}), 7.34(\mathrm{~d}, J=4.4 \mathrm{~Hz}$, $4 \mathrm{H}), 7.30 \sim 7.29(\mathrm{~m}, 2 \mathrm{H}), 5.26(\mathrm{~s}, 2 \mathrm{H}), 4.03(\mathrm{~s}, 2 \mathrm{H}) ;{ }^{13} \mathrm{C}$ NMR $\left(100 \mathrm{MHz}, \mathrm{CDCl}_{3}\right) \delta: 161.8,136.4,136.3,135.1$, $132.1,128.9,128.6,128.0,127.9,126.9,126.2,123.6$, 108.8, 99.3, 52.4, 51.8. HRMS (ESI) calcd for $\mathrm{C}_{18} \mathrm{H}_{15} \mathrm{Cl}_{3} \mathrm{NO}[\mathrm{M}+\mathrm{H}]^{+}$366.0214, found 366.0216.

2-乙基-4-(2,2,2-三氯乙基)异喹啉-1(2H)-酮(2j): 黄 色固体, 产率 33\%. m.p. 95 96 ${ }^{\circ} \mathrm{C} ;{ }^{1} \mathrm{H}$ NMR $(400 \mathrm{MHz}$, $\left.\mathrm{CDCl}_{3}\right) \delta: 8.50(\mathrm{dd}, J=8.0,1.2 \mathrm{~Hz}, 1 \mathrm{H}), 7.83(\mathrm{~d}, J=8.4$ $\mathrm{Hz}, 1 \mathrm{H}), 7.71 \sim 7.67(\mathrm{~m}, 1 \mathrm{H}), 7.53 \sim 7.49(\mathrm{~m}, 1 \mathrm{H}), 7.31(\mathrm{~s}$, $1 \mathrm{H}), 4.12$ (t, $J=7.2 \mathrm{~Hz}, 2 \mathrm{H}), 4.08$ (s, 2H), 1.41 (t, $J=7.2$ $\mathrm{Hz}, 3 \mathrm{H}) ;{ }^{13} \mathrm{C}$ NMR $\left(100 \mathrm{MHz}, \mathrm{CDCl}_{3}\right) \delta: 161.4,136.4$, 135.0, 131.9, 128.3, 126.8, 126.2, 123.5, 108.6, 99.4, 52.5, 44.4, 14.7. HRMS (ESI) calcd for $\mathrm{C}_{13} \mathrm{H}_{13} \mathrm{Cl}_{3} \mathrm{NO}[\mathrm{M}+\mathrm{H}]^{+}$ 304.0057 , found 304.0057 .

2-乙基-4-(2,2,2-三氯乙基)-3,4-二氢异喹啉-1(2H)酮 (2j'): 无色液体, 产率 $44 \% .{ }^{1} \mathrm{H}$ NMR $(400 \mathrm{MHz}$, $\left.\mathrm{CDCl}_{3}\right) \delta: 8.10(\mathrm{dd}, J=8.0,1.2 \mathrm{~Hz}, 1 \mathrm{H}), 7.47$ (td, $J=7.6$, $1.2 \mathrm{~Hz}, 1 \mathrm{H}), 7.39(\mathrm{td}, J=7.6,0.8 \mathrm{~Hz}, 1 \mathrm{H}), 7.33(\mathrm{~d}, J=7.6$
$\mathrm{Hz}, 1 \mathrm{H}), 3.90$ (dd, $J=13.2,4.0 \mathrm{~Hz}, 1 \mathrm{H}), 3.78$ (dd, $J=12.8$, $2.4 \mathrm{~Hz}, 1 \mathrm{H}), 3.74 \sim 3.67(\mathrm{~m}, 1 \mathrm{H}), 3.66 \sim 3.59(\mathrm{~m}, 1 \mathrm{H})$, $3.49 \sim 3.45(\mathrm{~m}, 1 \mathrm{H}), 3.24(\mathrm{dd}, J=15.2,7.6 \mathrm{~Hz}, 1 \mathrm{H}), 2.85$ (dd, $J=15.6,2.8 \mathrm{~Hz}, 1 \mathrm{H}), 1.25$ (t, $J=7.2 \mathrm{~Hz}, 3 \mathrm{H}) ;{ }^{13} \mathrm{C}$ NMR $\left(100 \mathrm{MHz}, \mathrm{CDCl}_{3}\right) \delta: 163.9,140.4,132.2,128.9$, 128.6, 127.9, 126.5, 98.6, 57.0, 49.5, 42.3, 36.3, 12.6. HRMS (ESI) calcd for $\mathrm{C}_{13} \mathrm{H}_{15} \mathrm{Cl}_{3} \mathrm{NO}[\mathrm{M}+\mathrm{H}]^{+} 306.0214$, found 306.0212 .

2-正丙基-4-(2,2,2-三氯乙基)异喹啉-1(2H)-酮(2k): 黄色液体, 产率 $72 \% .{ }^{1} \mathrm{H}$ NMR $\left(400 \mathrm{MHz}, \mathrm{CDCl}_{3}\right) \delta: 8.50$ (dd, $J=8.0,0.8 \mathrm{~Hz}, 1 \mathrm{H}), 7.83(\mathrm{~d}, J=8.0 \mathrm{~Hz}, 1 \mathrm{H}), 7.71 \sim$ $7.67(\mathrm{~m}, 1 \mathrm{H}), 7.53 \sim 7.49(\mathrm{~m}, 1 \mathrm{H}), 7.30(\mathrm{~s}, 1 \mathrm{H}), 4.07(\mathrm{~s}$, $2 \mathrm{H}), 4.01(\mathrm{t}, J=7.2 \mathrm{~Hz}, 2 \mathrm{H}), 1.84(\mathrm{dd}, J=14.8,7.6 \mathrm{~Hz}$, $2 \mathrm{H}), 1.00(\mathrm{t}, J=7.6 \mathrm{~Hz}, 3 \mathrm{H}) ;{ }^{13} \mathrm{C}$ NMR $\left(100 \mathrm{MHz}, \mathrm{CDCl}_{3}\right)$ $\delta: 161.6,136.4,135.6,131.9,128.4,126.8,126.2,123.4$, 108.3, 99.5, 52.5, 51.1, 22.5, 11.2. HRMS (ESI) calcd for $\mathrm{C}_{14} \mathrm{H}_{15} \mathrm{Cl}_{3} \mathrm{NO}[\mathrm{M}+\mathrm{H}]^{+}$318.0214, found 318.0213.

4-(2,2-二氯乙基)-2-甲基-3,4-二氢异喹啉-1(2H)-酮 (3a): 无色液体, 产率 $70 \% .{ }^{1} \mathrm{H}$ NMR $\left(600 \mathrm{MHz}, \mathrm{CDCl}_{3}\right)$ $\delta: 8.12(\mathrm{~d}, J=7.8 \mathrm{~Hz}, 1 \mathrm{H}), 7.47$ (td, $J=7.8,1.2 \mathrm{~Hz}, 1 \mathrm{H})$, $7.42 \sim 7.40(\mathrm{~m}, 1 \mathrm{H}), 7.27(\mathrm{~s}, 1 \mathrm{H}), 5.53(\mathrm{dd}, J=8.4,4.2 \mathrm{~Hz}$, $1 \mathrm{H}), 3.94$ (dd, $J=12.6,4.2 \mathrm{~Hz}, 1 \mathrm{H}), 3.33(\mathrm{dd}, J=13.2,1.8$ $\mathrm{Hz}, 1 \mathrm{H}), 3.24 \sim 3.23(\mathrm{~m}, 1 \mathrm{H}), 3.18(\mathrm{~s}, 3 \mathrm{H}), 2.61 \sim 2.56(\mathrm{~m}$, 1H), $2.54 \sim 2.50(\mathrm{~m}, 1 \mathrm{H}) ;{ }^{13} \mathrm{C} \mathrm{NMR}\left(150 \mathrm{MHz}, \mathrm{CDCl}_{3}\right) \delta$ : 164.1, 139.1, 131.9, 128.9, 128.6, 128.0, 126.6, 71.3, 52.5, 47.0, 35.5, 35.3. HRMS (ESI) calcd for $\mathrm{C}_{12} \mathrm{H}_{14} \mathrm{Cl}_{2} \mathrm{NO}$ $[\mathrm{M}+\mathrm{H}]^{+}$258.0447, found 258.0443 .

4-(2,2-二氯乙基)-2,6-二甲基-3,4-二氢异喹啉1 $(2 \mathrm{H})$-酮 (3b): 黄色液体, 产率 70\%. ${ }^{1} \mathrm{H}$ NMR $(400 \mathrm{MHz}$, $\left.\mathrm{CDCl}_{3}\right) \delta: 7.99(\mathrm{~d}, J=8.0 \mathrm{~Hz}, 1 \mathrm{H}), 7.20(\mathrm{~d}, J=7.6 \mathrm{~Hz}$, $1 \mathrm{H}), 7.04(\mathrm{~s}, 1 \mathrm{H}), 5.52(\mathrm{dd}, J=8.4,4.8 \mathrm{~Hz}, 1 \mathrm{H}), 3.90(\mathrm{dd}$, $J=12.8,4.4 \mathrm{~Hz}, 1 \mathrm{H}$ ), 3.29 (dd, $J=12.8,1.6 \mathrm{~Hz}, 1 \mathrm{H}), 3.15$ (s, $4 \mathrm{H}), 2.60 \sim 2.54(\mathrm{~m}, 1 \mathrm{H}), 2.53 \sim 2.49(\mathrm{~m}, 1 \mathrm{H}), 2.40(\mathrm{~s}$, $3 \mathrm{H}) ;{ }^{13} \mathrm{C}$ NMR $\left(100 \mathrm{MHz}, \mathrm{CDCl}_{3}\right) \delta: 164.2,142.5,139.1$, 129.0, 128.9, 127.1, 125.9, 71.4, 52.6, 47.0, 35.4, 35.3, 21.6. HRMS (ESI) calcd for $\mathrm{C}_{13} \mathrm{H}_{16} \mathrm{Cl}_{2} \mathrm{NO}[\mathrm{M}+\mathrm{H}]^{+}$ 272.0603 , found 272.0608 .

4-(2,2-二氯乙基)-6-甲氧基-2-甲基-3,4-二氢异喹啉1 $(2 H)$-酮(3c): 黄色液体, 产率 $42 \% .{ }^{1} \mathrm{H}$ NMR $(600 \mathrm{MHz}$, $\left.\mathrm{CDCl}_{3}\right) \delta: 8.04(\mathrm{~d}, J=8.4 \mathrm{~Hz}, 1 \mathrm{H}), 6.88(\mathrm{dd}, J=8.4,2.4$ $\mathrm{Hz}, 1 \mathrm{H}), 6.74(\mathrm{~d}, J=1.8 \mathrm{~Hz}, 1 \mathrm{H}), 5.53(\mathrm{dd}, J=9.0,4.8 \mathrm{~Hz}$, $1 \mathrm{H}), 3.89(\mathrm{dd}, J=13.2,4.2 \mathrm{~Hz}, 1 \mathrm{H}), 3.84(\mathrm{~s}, 3 \mathrm{H}), 3.28(\mathrm{dd}$, $J=12.6,1.8 \mathrm{~Hz}, 1 \mathrm{H}), 3.16 \sim 3.14(\mathrm{~m}, 1 \mathrm{H}), 3.13(\mathrm{~s}, 3 \mathrm{H})$, $2.59 \sim 2.54(\mathrm{~m}, 1 \mathrm{H}), 2.51 \sim 2.46(\mathrm{~m}, 1 \mathrm{H}) ;{ }^{13} \mathrm{C} \mathrm{NMR}(150$ $\left.\mathrm{MHz}, \mathrm{CDCl}_{3}\right) \delta: 164.1,162.3,141.0,131.0,121.3,113.1$, 
111.9, 71.4, 55.4, 52.5, 46.9, 35.6, 35.3. HRMS (ESI) calcd for $\mathrm{C}_{13} \mathrm{H}_{16} \mathrm{Cl}_{2} \mathrm{NO}_{2}[\mathrm{M}+\mathrm{H}]^{+}$288.0553, found 288.0558.

4-(2,2-二氯乙基)-6-氟-2-甲基-3,4-二氢异喹啉1(2H)-酮(3d): 黄色液体, 产率 46\%. ${ }^{1} \mathrm{H}$ NMR (400 MHz, $\left.\mathrm{CDCl}_{3}\right) \delta: 8.12(\mathrm{dd}, J=8.4,5.6 \mathrm{~Hz}, 1 \mathrm{H}), 7.07$ (td, $J=8.8$, $2.8 \mathrm{~Hz}, 1 \mathrm{H}), 6.96(\mathrm{dd}, J=8.8,2.4 \mathrm{~Hz}, 1 \mathrm{H}), 5.54(\mathrm{dd}, J=$ 8.0, $5.2 \mathrm{~Hz}, 1 \mathrm{H}), 3.92$ (dd, $J=13.2,4.4 \mathrm{~Hz}, 1 \mathrm{H}), 3.32$ (dd, $J=12.8,2.4 \mathrm{~Hz}, 1 \mathrm{H}), 3.24 \sim 3.19(\mathrm{~m}, 1 \mathrm{H}), 3.15(\mathrm{~s}, 3 \mathrm{H})$, $2.61 \sim 2.55(\mathrm{~m}, 1 \mathrm{H}), 2.54 \sim 2.48(\mathrm{~m}, 1 \mathrm{H}) ;{ }^{13} \mathrm{C}$ NMR $(100$ $\left.\mathrm{MHz}, \mathrm{CDCl}_{3}\right) \delta: 165.8,163.3(\mathrm{~d}, J=1.7 \mathrm{~Hz}), 141.8(\mathrm{~d}, J=$ $8.4 \mathrm{~Hz}), 131.7$ (d, $J=9.4 \mathrm{~Hz}), 124.9$ (d, $J=3.2 \mathrm{~Hz}), 115.2$ (d, $J=21.6 \mathrm{~Hz}), 113.5$ (d, $J=22.1 \mathrm{~Hz}), 71.0,52.3,46.6$, $35.4,35.3(\mathrm{~d}, J=1.7 \mathrm{~Hz}) ;{ }^{19} \mathrm{~F}$ NMR $\left(565 \mathrm{MHz}, \mathrm{CDCl}_{3}\right) \delta$ : -106.62 (s, 1F). HRMS (ESI) calcd for $\mathrm{C}_{12} \mathrm{H}_{13} \mathrm{Cl}_{2} \mathrm{FNO}$ $[\mathrm{M}+\mathrm{H}]^{+}$276.0353, found 276.0358 .

6-氯-4-(2,2-二氯乙基)-2-甲基-3,4-二氢异喹啉1(2H)-酮(3e): 黄色液体, 产率 $65 \% .{ }^{1} \mathrm{H} \mathrm{NMR}(400 \mathrm{MHz}$, $\left.\mathrm{CDCl}_{3}\right) \delta: 8.06(\mathrm{~d}, J=8.4 \mathrm{~Hz}, 1 \mathrm{H}), 7.38(\mathrm{dd}, J=8.4,2.0$ $\mathrm{Hz}, 1 \mathrm{H}), 7.27$ (d, $J=3.6 \mathrm{~Hz}, 1 \mathrm{H}), 5.56(\mathrm{dd}, J=8.0,5.2 \mathrm{~Hz}$, $1 \mathrm{H}), 3.92(\mathrm{dd}, J=12.8,4.0 \mathrm{~Hz}, 1 \mathrm{H}), 3.34(\mathrm{dd}, J=12.8,2.0$ $\mathrm{Hz}, 1 \mathrm{H}), 3.23 \sim 3.22(\mathrm{~m}, 1 \mathrm{H}), 3.17(\mathrm{~s}, 3 \mathrm{H}), 2.61 \sim 2.56(\mathrm{~m}$, 1H), $2.54 \sim 2.49(\mathrm{~m}, 1 \mathrm{H}) ;{ }^{13} \mathrm{C}$ NMR $\left(100 \mathrm{MHz}, \mathrm{CDCl}_{3}\right) \delta$ : $163.3,140.8,138.0,130.5,128.4,127.0,126.6,71.0,52.2$, 46.6, 35.5, 35.1. HRMS (ESI) calcd for $\mathrm{C}_{12} \mathrm{H}_{13} \mathrm{Cl}_{3} \mathrm{NO}$ $[\mathrm{M}+\mathrm{H}]^{+}$292.0057, found 292.0062.

6-溴-4-(2,2-二氯乙基)-2-甲基-3,4-二氢异喹啉1(2H)-酮(3f): 无色液体, 产率 50\%. ${ }^{1} \mathrm{H} \mathrm{NMR}(400 \mathrm{MHz}$, $\left.\mathrm{CDCl}_{3}\right) \delta: 7.97(\mathrm{~d}, J=8.4 \mathrm{~Hz}, 1 \mathrm{H}), 7.54(\mathrm{dd}, J=8.4,1.6$ $\mathrm{Hz}, 1 \mathrm{H}), 7.41$ (d, $J=1.6 \mathrm{~Hz}, 1 \mathrm{H}), 5.55$ (dd, $J=8.0,5.2 \mathrm{~Hz}$, $1 \mathrm{H}), 3.91(\mathrm{dd}, J=12.8,4.0 \mathrm{~Hz}, 1 \mathrm{H}), 3.33(\mathrm{dd}, J=13.2,2.4$ $\mathrm{Hz}, 1 \mathrm{H}), 3.22 \sim 3.20(\mathrm{~m}, 1 \mathrm{H}), 3.16(\mathrm{~s}, 3 \mathrm{H}), 2.60 \sim 2.54(\mathrm{~m}$, $1 \mathrm{H}), 2.53 \sim 2.48(\mathrm{~m}, 1 \mathrm{H}) ;{ }^{13} \mathrm{C} \mathrm{NMR}\left(100 \mathrm{MHz}, \mathrm{CDCl}_{3}\right) \delta$ : $163.4,141.0,131.4,130.7,129.5,127.5,126.6,71.0,52.2$, 46.6, 35.5, 35.0. HRMS (ESI) calcd for $\mathrm{C}_{12} \mathrm{H}_{13} \mathrm{BrCl}_{2} \mathrm{NO}$ $[\mathrm{M}+\mathrm{H}]^{+} 337.9529$, found 337.9528 .

4-(2,2-二氯乙基)-6-碘-2-甲基-3,4-二氢异喹啉1(2H)-酮(3g): 无色液体, 产率 55\%. ${ }^{1} \mathrm{H} \mathrm{NMR}(400 \mathrm{MHz}$, $\left.\mathrm{CDCl}_{3}\right) \delta: 7.81(\mathrm{~d}, J=8.0 \mathrm{~Hz}, 1 \mathrm{H}), 7.76(\mathrm{~d}, J=8.4 \mathrm{~Hz}$, $1 \mathrm{H}), 7.62$ (s, 1H), 5.55 (dd, $J=7.6,5.6 \mathrm{~Hz}, 1 \mathrm{H}), 3.90$ (dd, $J=12.8,4.0 \mathrm{~Hz}, 1 \mathrm{H}), 3.32(\mathrm{dd}, J=12.8,2.0 \mathrm{~Hz}, 1 \mathrm{H})$, $3.21 \sim 3.18(\mathrm{~m}, 1 \mathrm{H}), 3.15(\mathrm{~s}, 3 \mathrm{H}), 2.55 \sim 2.53(\mathrm{~m}, 1 \mathrm{H})$, $2.53 \sim 2.51(\mathrm{~m}, 1 \mathrm{H}) ;{ }^{13} \mathrm{C}$ NMR $\left(100 \mathrm{MHz}, \mathrm{CDCl}_{3}\right) \delta$ : $163.6,141.0,137.4,135.4,130.5,128.1,99.2,71.0,52.2$, 46.6, 35.5, 34.9. HRMS (ESI) calcd for $\mathrm{C}_{12} \mathrm{H}_{13} \mathrm{Cl}_{2} \mathrm{INO}$ $[\mathrm{M}+\mathrm{H}]^{+}$383.9413, found 383.9421 .
2-芐基-4-(2,2-二氯乙基)-3,4-二氢异喹啉-1(2H)酮(3h): 无色液体, 产率 60\%. ${ }^{1} \mathrm{H}$ NMR (400 MHz, $\left.\mathrm{CDCl}_{3}\right) \delta: 8.18(\mathrm{~d}, J=7.6 \mathrm{~Hz}, 1 \mathrm{H}), 7.47 \sim 7.42(\mathrm{~m}, 2 \mathrm{H})$, $7.38 \sim 7.34(\mathrm{~m}, 5 \mathrm{H}), 7.22(\mathrm{~d}, J=7.2 \mathrm{~Hz}, 1 \mathrm{H}), 5.24(\mathrm{~d}, J=$ $14.4 \mathrm{~Hz}, 1 \mathrm{H}), 5.03$ (t, $J=7.2 \mathrm{~Hz}, 1 \mathrm{H}), 4.30$ (d, $J=14.4 \mathrm{~Hz}$, $1 \mathrm{H}), 3.76(\mathrm{dd}, J=12.8,4.0 \mathrm{~Hz}, 1 \mathrm{H}), 3.24$ (dd, $J=12.8,1.2$ $\mathrm{Hz}, 1 \mathrm{H}), 3.14 \sim 3.10(\mathrm{~m}, 1 \mathrm{H}), 2.32 \sim 2.26(\mathrm{~m}, 1 \mathrm{H}), 2.20 \sim$ $2.13(\mathrm{~m}, 1 \mathrm{H}) .{ }^{13} \mathrm{C} \mathrm{NMR}\left(150 \mathrm{MHz}, \mathrm{CDCl}_{3}\right) \delta: 163.7$, $139.4,136.8,132.1,129.2,128.9,128.7,128.0,127.9$, 126.6, 70.9, 50.2, 48.1, 46.8, 35.1. HRMS (ESI) calcd for $\mathrm{C}_{18} \mathrm{H}_{18} \mathrm{Cl}_{2} \mathrm{NO}[\mathrm{M}+\mathrm{H}]^{+} 334.0760$, found 334.0757.

4-(2,2-二氯乙基)-2-乙基-3,4-二氢异喹啉-1(2H)酮(3i): 黄色液体, 产率 $80 \%$. ${ }^{1} \mathrm{H}$ NMR (400 $\mathrm{MHz}$, $\left.\mathrm{CDCl}_{3}\right) \delta: 8.13(\mathrm{~d}, J=7.6 \mathrm{~Hz}, 1 \mathrm{H}), 7.50 \sim 7.45(\mathrm{~m}, 1 \mathrm{H})$, $7.43 \sim 7.40(\mathrm{~m}, 1 \mathrm{H}), 7.28(\mathrm{~s}, 1 \mathrm{H}), 5.51(\mathrm{dd}, J=8.4,4.4 \mathrm{~Hz}$, $1 \mathrm{H}), 3.92$ (dd, $J=12.8,4.0 \mathrm{~Hz}, 1 \mathrm{H}), 3.70(\mathrm{dd}, J=13.6,6.8$ $\mathrm{Hz}, 1 \mathrm{H}), 3.59$ (dd, $J=13.6,7.2 \mathrm{~Hz}, 1 \mathrm{H}), 3.34$ (dd, $J=13.2$, $2.0 \mathrm{~Hz}, 1 \mathrm{H}), 3.26 \sim 3.21(\mathrm{~m}, 1 \mathrm{H}), 2.60 \sim 2.53(\mathrm{~m}, 1 \mathrm{H})$, $2.52 \sim 2.46(\mathrm{~m}, 1 \mathrm{H}), 1.24(\mathrm{t}, J=7.2 \mathrm{~Hz}, 3 \mathrm{H}) ;{ }^{13} \mathrm{C} \mathrm{NMR}$ $\left(150 \mathrm{MHz}, \mathrm{CDCl}_{3}\right) \delta: 163.4,138.9,131.8,128.9,128.8$, 128.0, 126.5, 71.3, 49.9, 46.9, 42.1, 35.1, 12.5. HRMS (ESI) calcd for $\mathrm{C}_{13} \mathrm{H}_{16} \mathrm{Cl}_{2} \mathrm{NO}[\mathrm{M}+\mathrm{H}]^{+}$272.0603, found 272.0601 .

4-(2,2-二氯乙基)-2-正己基-3,4-二氢异喹啉-1(2H)酩 (3j): 无色液体，产率 $72 \%$. ${ }^{1} \mathrm{H}$ NMR $(600 \mathrm{MHz}$, $\left.\mathrm{CDCl}_{3}\right) \delta: 8.13(\mathrm{~d}, J=7.8 \mathrm{~Hz}, 1 \mathrm{H}), 7.47(\mathrm{dt}, J=7.8,1.2$ $\mathrm{Hz}, 1 \mathrm{H}), 7.41(\mathrm{t}, J=6.6 \mathrm{~Hz}, 1 \mathrm{H}), 7.27(\mathrm{~s}, 1 \mathrm{H}), 5.52$ (dd, $J=9.0,4.8 \mathrm{~Hz}, 1 \mathrm{H}), 3.90(\mathrm{dd}, J=12.6,4.2 \mathrm{~Hz}, 1 \mathrm{H}), 3.60$ (dd, $J=13.2,7.8 \mathrm{~Hz}, 1 \mathrm{H}), 3.56$ (dd, $J=14.4,7.8 \mathrm{~Hz}, 1 \mathrm{H}$ ), $3.34(\mathrm{dd}, J=13.2,1.8 \mathrm{~Hz}, 1 \mathrm{H}), 3.24 \sim 3.23(\mathrm{~m}, 1 \mathrm{H})$, $2.59 \sim 2.55(\mathrm{~m}, 1 \mathrm{H}), 2.52 \sim 2.49(\mathrm{~m}, 1 \mathrm{H}), 1.64 \sim 1.60(\mathrm{~m}$, $3 \mathrm{H}), 1.35 \sim 1.32(\mathrm{~m}, 5 \mathrm{H}), 0.90(\mathrm{t}, J=6.6 \mathrm{~Hz}, 3 \mathrm{H}) ;{ }^{13} \mathrm{C}$ NMR $\left(150 \mathrm{MHz}, \mathrm{CDCl}_{3}\right) \delta: 163.6,139.0,131.8,129.1$, $128.9,128.1,126.5,71.3,50.4,47.6,47.1,35.3,31.6,27.6$, 26.7, 22.5, 14.0. HRMS (ESI) calcd for $\mathrm{C}_{17} \mathrm{H}_{24} \mathrm{Cl}_{2} \mathrm{NO}$ $[\mathrm{M}+\mathrm{H}]^{+}$328.1229, found 328.1225 .

2-环戊基-4-(2,2-二氯乙基)-3,4-二氢异喹啉-1(2H)酮(3k): 黄色液体, 产率 $60 \% .{ }^{1} \mathrm{H}$ NMR $(600 \mathrm{MHz}$, $\left.\mathrm{CDCl}_{3}\right) \delta: 8.13(\mathrm{~d}, J=7.2 \mathrm{~Hz}, 1 \mathrm{H}), 7.46(\mathrm{dt}, J=6.6,0.6$ $\mathrm{Hz}, 1 \mathrm{H}), 7.40$ (t, $J=7.2 \mathrm{~Hz}, 1 \mathrm{H}), 7.27$ (s, 1H), 5.50 (dd, $J=9.0,4.2 \mathrm{~Hz}, 1 \mathrm{H}), 5.22 \sim 5.19(\mathrm{~m}, 1 \mathrm{H}), 3.69$ (dd, $J=$ 12.6, $3.6 \mathrm{~Hz}, 1 \mathrm{H}), 3.32$ (dd, $J=13.2,2.4 \mathrm{~Hz}, 1 \mathrm{H}), 3.25 \sim$ $3.23(\mathrm{~m}, 1 \mathrm{H}), 2.54 \sim 2.49(\mathrm{~m}, 1 \mathrm{H}), 2.47 \sim 2.43(\mathrm{~m}, 1 \mathrm{H})$, $1.99 \sim 1.95(\mathrm{~m}, 1 \mathrm{H}), 1.88 \sim 1.86(\mathrm{~m}, 1 \mathrm{H}), 1.76 \sim 1.72(\mathrm{~m}$, $2 \mathrm{H}), 1.69 \sim 1.65(\mathrm{~m}, 2 \mathrm{H}), 1.53 \sim 1.49(\mathrm{~m}, 2 \mathrm{H}) ;{ }^{13} \mathrm{C} \mathrm{NMR}$ 
$\left(150 \mathrm{MHz}, \mathrm{CDCl}_{3}\right) \delta: 163.6,138.7,131.8,129.3,129.2$, 128.1, 126.5, 71.4, 53.8, 46.7, 44.7, 35.1, 29.0, 27.8, 24.2, 24.1. HRMS calcd for $\mathrm{C}_{16} \mathrm{H}_{20} \mathrm{Cl}_{2} \mathrm{NO}[\mathrm{M}+\mathrm{H}]^{+} 312.0916$, found 312.0913 .

3-甲基-1-对甲苯磺酰基-4-(2,2,2-三氯乙基)吡咯烷 (4a-1): 无色液体，产率大于 $99 \%(2.9 / 1) .{ }^{1} \mathrm{H}$ NMR (400 $\left.\mathrm{MHz}, \mathrm{CDCl}_{3}\right) \delta: 7.73(\mathrm{~d}, J=8.0 \mathrm{~Hz}, 2 \mathrm{H}), 7.34(\mathrm{~d}, J=8.0$ $\mathrm{Hz}, 2 \mathrm{H}), 3.67$ (dd, $J=10.0,6.8 \mathrm{~Hz}, 1 \mathrm{H}), 3.37$ (dd, $J=10.0$, $6.4 \mathrm{~Hz}, 1 \mathrm{H}), 3.18$ (dd, $J=10.0,8.4 \mathrm{~Hz}, 1 \mathrm{H}), 3.07$ (dd, $J=$ 10.0, $2.8 \mathrm{~Hz}, 1 \mathrm{H}$ ), 2.78 (dd, $J=18.4,7.2 \mathrm{~Hz}, 1 \mathrm{H}$ ), 2.48 (d, $J=10.0 \mathrm{~Hz}, 2 \mathrm{H}), 2.44(\mathrm{~s}, 3 \mathrm{H}), 2.40 \sim 2.31(\mathrm{~m}, 1 \mathrm{H}), 0.80$ $(\mathrm{d}, J=7.2 \mathrm{~Hz}, 3 \mathrm{H}) ;{ }^{13} \mathrm{C} \mathrm{NMR}\left(100 \mathrm{MHz}, \mathrm{CDCl}_{3}\right) \delta: 143.5$, 133.8, 129.7, 127.3, 98.7, 54.3, 53.5, 51.0, 39.7, 35.9, 21.5, 13.5. HRMS calcd for $\mathrm{C}_{14} \mathrm{H}_{19} \mathrm{Cl}_{3} \mathrm{NO}_{2} \mathrm{~S}[\mathrm{M}+\mathrm{H}]{ }^{+}$ 370.0197, found 370.0193.

3-甲基-1-对甲苯磺酰基-4-(2,2,2-三氯乙基)吡咯烷 (4a-2): 无色液体，产率大于 $99 \%$ (2.9/1). ${ }^{1} \mathrm{H}$ NMR (400 $\left.\mathrm{MHz} \mathrm{CDCl}_{3}\right) \delta: 7.72(\mathrm{~d}, J=8.0 \mathrm{~Hz}, 2 \mathrm{H}), 7.34(\mathrm{~d}, J=8.0$ $\mathrm{Hz}, 2 \mathrm{H}), 3.85$ (dd, $J=10.4,7.6 \mathrm{~Hz}, 1 \mathrm{H}), 3.53$ (dd, $J=10.0$, $7.6 \mathrm{~Hz}, 1 \mathrm{H}), 2.83(\mathrm{dd}, J=14.8,2.0 \mathrm{~Hz}, 1 \mathrm{H}), 2.80 \sim 2.75$ $(\mathrm{m}, 1 \mathrm{H}), 2.59(\mathrm{dd}, J=14.2,9.6 \mathrm{~Hz}, 1 \mathrm{H}), 2.51 \sim 2.46(\mathrm{~m}$, $1 \mathrm{H}), 2.44(\mathrm{~s}, 3 \mathrm{H}), 2.02 \sim 1.94(\mathrm{~m}, 1 \mathrm{H}), 1.86 \sim 1.77(\mathrm{~m}$, $1 \mathrm{H}), 1.00(\mathrm{~d}, J=6.4 \mathrm{~Hz}, 3 \mathrm{H}) ;{ }^{13} \mathrm{C} \mathrm{NMR}\left(100 \mathrm{MHz}, \mathrm{CDCl}_{3}\right)$ $\delta$ : 143.6, 133.5, 129.7, 127.4, 98.4, 57.3, 53.8, 53.5, 43.5, $38.8,21.5,15.5$.

致谢 感谢河北大学高性能计算中心提供的理论计算 数据.

辅助材料(Supporting Information) 产物 2a 的核磁 共振氢谱、碳谱和高分辨质谱. 这些材料可以免费从 本刊网站(http://sioc-journal.cn/)上下载

\section{References}

[1] For selected recent reviews, see:

(a) Gribble, G. W. Acc. Chem. Res. 1998, 31, 141.

(b) Vaillancourt, F. H.; Yeh, E.; Vosburg, D. A.; Garneau-Tsodikova,

S.; Walsh, C. T. Chem. Rev. 2006, 106, 3364

(c) Paul, C.; Pohnert, G. Nat. Prod. Rep. 2011, 28, 186.

(d) Latham, J.; Brandenburger, E.; Shepherd, S. A.; Menon, B. R. K.; Micklefield, J. Chem. Rev. 2018, 118, 232.

[2] For selected recent reviews, see:

(a) Sitachitta, N.; Rossi, J.; Roberts, M. A.; Gerwick, W. H.; Fletcher, M. D.; Willis, C. L. J. Am. Chem. Soc. 1998, 120, 7131.

(b) Ardá, A.; Soengas, R. G.; Nieto, M. I.; Jiménez, C.; Rodríguez, J. Org. Lett. 2008, 10, 2175.

(c) Nguyen, V.-A.; Willis, C. L.; Gerwick, W. H. Chem. Commun. 2001, 1934.

[3] For selected recent references, see:

(a) Butler, A.; Walker, J. V. Chem. Rev. 1993, 93, 1937.

(b) Morimoto, H.; Lu, G.; Aoyama, N.; Matsunaga, S.; Shibasaki,
M. J. Am. Chem. Soc. 2007, 129,9588.

(c) Miles, Z. D.; Diethelm, S.; Pepper, H. P.; Huang, D. M.; George, J. H.; Moore, B. S. Nat. Chem. 2017, 9, 1235.

[4] For selected recent reviews, see:

(a) Vaillancourt, F. H.; Yeh, E.; Vosburg, D. A.; O'Connor, S. E.; Walsh, C. T. Nature 2005, 436, 1191.

(b) Galonić, D. P.; Vaillancourt, F. H.; Walsh, C. T. J. Am. Chem. Soc. 2006, 128, 3900.

[5] For selected recent reviews, see:

(a) Beaumont, S.; Ilardi, E. A.; Monroe, L. R.; Zakarian, A. J. Am. Chem. Soc. 2010, 132, 1482.

(b) Gu, Z. H.; Herrmann, A. T.; Zakarian, A. Angew. Chem., Int. Ed 2011, 50, 7136.

[6] Lu, M. Z.; Loh, T.-P. Org. Lett. 2014, 16, 4698.

[7] Li, X.; Xu, J.; Gao, Y.; Fang, H.; Tang, G.; Zhao, Y. J. Org. Chem. 2015, 80, 2621.

[8] For selected recent reviews on cyclization reactions, see: (a) Liu, Y.; Zhang, J.-L.; Song, R.-J.; Li, J.-H. Org. Chem. Front 2014, $1,1289$.

(b) Liang, Y.; Lv, G.; Ouyang, X.; Song, R.-J.; Li, J.-H. Adv. Synth. Catal. 2020, 363, 290.

(c) Tian, Y.; Liu, Z.-Q. RSC Adv. 2014, 4, 64855.

(d) Pan, C.; Wu, C.; Yuan, C.; Yu, J.-T. Tetrahedron Lett. 2020, 61, 151499 .

(e) Huang, G.; Yu, J.-T.; Pan, C.-D. Adv. Synth. Catal. 2020, 363, 305 .

(1) Li, W.; Sun, Y.; Yao, Y.; Xu, Y.; Li, P.; Liu, Y.; Liang, D. Chin. J. Org. Chem. 2019, 39, 1727 (in Chinese).

(李文兰, 孙一茼, 姚永超, 许颖, 李鹏, 刘颖杰, 梁德强, 有机 化学, 2019, 39, 1727.)

[9] For selected recent reviews, see:

(a) Pettit, G. R.; Gaddamidi, V.; Cragg, G. M.; Herald, D. L.; Sagawa, Y. J. Chem. Soc., Chem. Commun. 1984, 1692

(b) Pettit, G. R.; Meng, Y.; Herald, D. L.; Graham, K. A. N.; Pettit, R. K.; Doubek, D. L. J. Nat. Prod. 2003, 66, 1065.

(c) Krane, B. D.; Shamma, M. J. Nat. Prod. 1982, 45, 377.

(d) Cheng, K.; Rahier, N. J.; Eisenhauer, B. M.; Gao, R.; Thomas, S. J.; Hecht, S. M. J. Am. Chem. Soc. 2005, 127, 838.

[10] Scott, J. D.; Williams, R. M. Chem. Rev. 2002, 102, 1669.

[11] For selected recent reviews, see:

(a) Kajita, Y.; Matsubara, S.; Kurahashi, T. J. Am. Chem. Soc. 2008 130, 6058 .

(b) Ackermann, L.; Lygin, A. V.; Hofmann, N. Angew. Chem., Int. Ed. 2011, 50, 6379 .

(c) Antczak, M. I.; Ready, J. M. Chem. Sci. 2012, 3, 1450.

(d) Upadhyay, N. S.; Thorat, V. H.; Sato, R.; Annamalai, P.; Chuang, S.-C.; Cheng, C.-H. Green Chem. 2017, 19, 3219.

(e) Hédouin, J.; Carpentier, V.; Renard, R. M. Q.; Schneider, C.; Gillaizeau, I.; Hoarau, C. J. Org. Chem. 2019, 84, 10535. (f) Fang, Z.; Wang, Y.; Wang, Y. Org. Lett. 2019, 21, 434 (g) Lee, J.; Kim, H. Y.; Oh, K. Org. Lett. 2020, 22, 474.

[12] For selected recent reviews, see:

(a) Azizian, J.; Mohammadi, A. A.; Karimi, A. R.; Mohammadizadeh, M. R. J. Organomet. Chem. 2005, 70, 350.

(b) Vara, Y.; Bello, T.; Aldaba, E.; Arrieta, A.; Pizarro, J. L.; Arriortua, M. I.; Lopez, X.; Cossio, F. P. Org. Lett. 2008, 10, 4759.

(c) Wang, L.; Liu, J.; Tian, H.; Qian, C.; Sun, J. Adv. Synth. Catal. 2005, 347, 689.

[13] For selected recent reviews, see:

(a) Tang, Q.; Xia, D.; Jin, X.; Zhang, Q.; Sun, X.-Q.; Wang, C. J. Am. Chem. Soc. 2013, 135, 4628.

(b) Rakshit, S.; Grohmann, C.; Besset, T.; Glorius, F. J. Am. Chem. Soc. 2011, 133, 2350.

(c) Li, B.; Ma, J.; Wang, N.; Feng, H.; Xu, S.; Wang, B. Org. Lett. 2012, 14, 736.

(d) Zhang, S.-S.; Wu, J.-Q.; Liu, X.; Wang, H. ACS Catal. 2015, 5, 210 .

[14] For selected recent radical cyclization reactions, see: 
(a) Zhou, W.; Ni, S.; Mei, H.; Han, J.; Pan, Y. Org. Lett. 2015, 17, 2724.

(b) Xia, D.; Li, Y.; Miao, T.; Li, P.; Wang, L. Chem. Commun. 2016, $52,11559$.

(c) Wang, J.; Sun, K.; Chen, X.; Chen, T.; Liu, Y.; Qu, L.; Zhao, Y.; Yu, B. Org. Lett. 2019, 21, 1863.

(d) Zou, L.; Li, P.; Wang, B.; Wang, L. Green Chem. 2019, 21, 3362.

(e) Pan, C.-D.; Wang, Y.; Wu, C.; Yu, J.-T. Catal. Commun. 2019, 131, 105802 .

(f) Liu, X.; Qian, P.; Wang, Y.; Pan, Y. Org. Chem. Front. 2017, 4, 2370. (g) Xu, Z.-Q.; Wang, C.; Li, L.; Duan, L.; Li, Y.-M. J. Org. Chem. 2018, 83, 9718 .

[15] (a) Li, Z.-J.; Cui, X.; Niu, L.; Ren, Y.; Bian, M.; Yang, X.; Yang, B.; Yan, Q.; Zhao, J. Adv. Synth. Catal. 2017, 359, 246.

(b) Zhang, R.; Yu, H.; Li, Z.-J.; Yan, Q.; Li, P.; Wu, J.; Qi, J.; Jiang, M.; Sun, L. Adv. Synth. Catal. 2018, 360, 1384.

(c) Ge, Y.; Tian, Y.; Wu, J.; Yan, Q.; Zheng, L.; Ren, Y.; Zhao, J.; Li, Z.-J. Chem. Commun. 2020, 56, 12656.

(d) Wu, J.; Zong, Y.; Zhao, C.; Yan, Q.; Sun, L.; Li, Y.; Zhao, J.; Ge, Y.; Li, Z.-J. Org. Biomol. Chem. 2019, 17, 794.

[16] Kundu, G.; Sperger, T.; Rissanen, K.; Schoenebeck, F. Angew. Chem., Int. Ed. 2020, 59, 21930. 